\section{Carfilzomib, cyclophosphamide and dexamethasone for newly diagnosed, high-risk myeloma patients not eligible for transplant: a pooled analysis of two studies}

\author{
Roberto Mina, ${ }^{1}$ Francesca Bonello, ${ }^{1}$ Maria Teresa Petrucci, ${ }^{2}$ \\ Anna Marina Liberati, ${ }^{3}$ Concetta Conticello, ${ }^{4}$ Stelvio Ballanti, ${ }^{5}$ \\ Pellegrino Musto, ${ }^{6^{\circ}}$ Attilio Olivieri, ${ }^{7}$ Giulia Benevolo, ${ }^{8}$ Andrea Capra, ${ }^{1}$ \\ Milena Gilestro, ${ }^{1}$ Piero Galieni, ${ }^{9}$ Michele Cavo,${ }^{10}$ Agostina Siniscalchi, ${ }^{11}$ \\ Antonio Palumbo ${ }^{1}{ }^{\circ}$ Vittorio Montefusco, ${ }^{12}$ Gianluca Gaidano, ${ }^{13}$ \\ Paola Omedé, ${ }^{1}$ Mario Boccadoro ${ }^{1}$ and Sara Bringhen ${ }^{1}$
}

\begin{abstract}
${ }^{1}$ Myeloma Unit, Division of Hematology, University of Torino, Azienda OspedalieroUniversitaria Città della Salute e della Scienza di Torino, Torino; ${ }^{2}$ Division of Hematology, Department of Cellular Biotechnologies and Hematology, Sapienza University of Rome, Rome; ${ }^{3}$ Università degli Studi di Perugia, Struttura Complessa Universitaria Oncoematologia - Azienda Ospedaliera Santa Maria di Terni, Terni; ${ }^{4}$ Division of Hematology, AOU Policlinico-OVE, University of Catania, Catania; ${ }^{5}$ Reparto di Ematologia con TMO, Ospedale Santa Maria della Misericordia, Perugia; ${ }^{6}$ Hematology Unit, IRCCSCROB, Rionero in Vulture (PZ); ${ }^{7}$ Clinica di Ematologia, Università Politecnica delle Marche, Ancona; ${ }^{8}$ Hematology, Città della Salute e della Scienza, Turin; ${ }^{9}$ Division of Hematology, Ospedale “C. e G. Mazzoni", ASUR Marche-AV5, Ascoli Piceno; ${ }^{10 " S e r a ̀ g n o l i " ~ I n s t i t u t e ~ o f ~}$ Hematology, Bologna University School of Medicine, Bologna; ${ }^{11}$ UOC Ematologia, Ospedale S. Eugenio, ASLRM2, Rome; ${ }^{12}$ Hematology Department, Fondazione IRCCS Istituto Nazionale Tumori, Milano and ${ }^{13}$ Division of Hematology, Department of Translational Medicine, Università del Piemonte Orientale, Novara, Italy
\end{abstract}

'PM is currently at the Department of Emergency and Organ Transplantation, "Aldo Moro" University School of Medicine, Unit of Hematology and Stem Cell Transplantation, AOU Consorziale Policlinico, Bari.

${ }^{\circ} A P$ is currently a GlaxoSmithKline AG employee.
$\mathrm{D}$ espite remarkable advances in the treatment of multiple myeloma $(\mathrm{MM})$ in the last decades, the prognosis of patients harboring highrisk cytogenetic abnormalities remains dismal as compared to that of standard-risk patients. Proteasome inhibitors have been demonstrated to partially ameliorate the prognosis of high-risk patients. We pooled together data from two phase I/II trials on transplant-ineligible patients with MM receiving upfront carfilzomib cyclophosphamide and dexamethasone followed by carfilzomib maintenance. The aim of this analysis was to compare treatment outcomes in patients with standard-risk versus high-risk cytogenetic abnormalities detected by fluorescence in situ hybridization (FISH) analysis. High risk was defined by the presence of at least one chromosomal abnormality, including $t(4 ; 14)$, del17p and $t(14 ; 16)$. Overall, 94 patients were included in the analysis: 57 (61\%) in the standard-risk and 37 $(39 \%)$ in the high-risk group. Median follow-up was 38 months. In standard-risk versus high-risk patients, we observed similar progression-free survival (PFS) (3-year PFS: $52 \%$ vs. $43 \%$, respectively; $P=0.50$ ), overall survival (OS) (3-year OS: 78\% vs. 73\%; $P=0.38)$, and overall response rate $(88 \%$ vs. $95 \% ; P=0.47)$, with no statistical differences between the two groups. No difference in terms of PFS was observed between patients with or without del17p. Carfilzomib, used both as induction and maintenance agent for transplant-ineligible newly diagnosed $\mathrm{MM}$ patients, mitigated the poor prognosis carried by high-risk cytogenetics and resulted in similar PFS and $\mathrm{OS}$ as in standard-risk patients. (Registered at clinicaltrials.gov identifiers: NCT01857115 [IST-CAR-561] and NCT01346787 [IST-CAR-506].)
Haematologica 2021

Volume 106(4):1079-1085

\section{Correspondence:}

SARA BRINGHEN

sarabringhen@yahoo.com

Received: November 21, 2019.

Accepted: February 19, 2020.

Pre-published: February 27, 2020.

https://doi.org/10.3324/haematol.2019.243428

(C)2021 Ferrata Storti Foundation

Material published in Haematologica is covered by copyright. All rights are reserved to the Ferrata Storti Foundation. Use of published material is allowed under the following terms and conditions:

https://creativecommons.org/licenses/by-nc/4.0/legalcode. Copies of published material are allowed for personal or internal use. Sharing published material for non-commercial purposes is subject to the following conditions: https://creativecommons.ors/licenses/by-nc/4.0/legalcode, sect. 3. Reproducing and sharing published material for commercial purposes is not allowed without permission in writing from the publisher. 


\section{Introduction}

Multiple myeloma (MM) is a plasma cell dyscrasia with a heterogeneous prognosis ranging from a few years to over a decade, according to both disease-related factors (such as albumin and $\beta-2$ microglobulin levels, cytogenetic abnormalities [CA] or presence of extramedullary disease) and patient-related factors (age, comorbidities, frailty status). ${ }^{1-3}$ To date, one of the most powerful prognostic markers in $\mathrm{MM}$ is the presence of either primary (translocations) or secondary (deletions or amplifications) recurrent CA detected by fluorescence in situ hybridization (FISH). Deletions of chromosome 17p and TP53 have been reported in $5-20 \%$ of $\mathrm{MM}$ patients according to the cut-off adopted by laboratories and have been clearly associated with a dismal prognosis. ${ }^{4}$ Another adverse CA is $t(4 ; 14)$, which is carried by $12-15 \%$ of MM patients and leads to the deregulation of fibroblast growth factor receptor 3 (FGFR3) and multiple myeloma SET domain (MMSET). ${ }^{5,6}$ Eventually, the occurrence of $t(14 ; 16)$ has been associated to worse progression-free survival (PFS) and overall survival (OS) in a study published by the Mayo Clinic,' although some doubts have been cast by another study by Intergroupe Francophone du Myélome $(\text { IFM })^{8}$ and conflicting results have been thereafter reported even in patients treated in the novel agent era. The presence of at least one of these three abnormalities identifies a subgroup of patients at high risk of relapse and death. ${ }^{9}$

MM is mainly a disease of the elderly, with a median age at diagnosis of 69 years. ${ }^{10}$ Older patients are usually considered not eligible for high-dose chemotherapy and autologous stem cell transplantation (ASCT). In this patient population, the initial therapeutic approach includes either a triplet proteasome inhibitor (PI)-based regimen (bortezomib-melphalan-prednisone, VMP), a two-drug regimen containing an immunomodulatory agent (IMiD; lenalidomide-dexamethasone, Rd), or a combination of both a PI and an IMiD (bortezomiblenalidomide-dexamethasone, VRD). ${ }^{11}$ In the VISTA study that led to the approval of the VMP combination, the median PFS was 19.8 months in high-risk (HiR) patients by FISH and 23 months in standard-risk (SR) patients (HR:1.29). ${ }^{12,13}$ In the FIRST study, among patients receiving continuous $\mathrm{Rd}$, the median PFS was 8.4 months in HiR patients versus 31.1 in SR patients. ${ }^{14,15}$

Carfilzomib is a second-generation PI currently approved for relapsed and/or refractory (RR)MM patients. In the phase III ENDEAVOR trial comparing carfilzomib-dexamethasone (Kd) to bortezomib-dexamethasone (Vd), the PFS and OS advantage of Kd observed in the overall population was also retained in HiR patients (median PFS in HiR patients treated with Kd vs. Vd: 8.8 vs. 6.0 months; $P=0.007) \cdot{ }^{16}$ Similarly, in the phase III ASPIRE trial, the triplet carfilzomib-lenalidomide-dexamethasone (KRd) proved to be superior to $\mathrm{Rd}$ also in patients with HiR CA (median PFS in HiR patients treated with KRd vs. Rd: 23.1 vs. 13.9 months; $P=0.08) .{ }^{17}$ Taken together, these results suggest that carfilzomib-based regimens might at least partially overcome the negative impact of HiR cytogenetics in MM patients.

We previously published the results of two phase I/II trials showing that the combination carfilzomibcyclophosphamide-dexamethasone (KCyd), followed by carfilzomib maintenance, was effective and well tolerated in newly diagnosed (ND) elderly MM patients (NDMM). ${ }^{18,19}$ Here we report the results of a pooled analysis of patient data from the two trials aiming at evaluating the efficacy of a carfilzomib-based therapy in SR and HiR patients.

\section{Methods}

\section{Study design and treatment}

We pooled together data from two phase I/II (IST-CAR-561; clinicaltrials.gov identifier: NCT01857115) and phase II (IST-CAR506; clinicaltrials.gov identifier: NCT01346787) studies. Both trials enrolled NDMM patients over 65 years of age or younger but not eligible for ASCT. Ethics committees or institutional review boards at the study sites approved both studies, which were carried out in accordance with the Declaration of Helsinki. All patients provided written informed consent.

Details of study procedures have been published previously. ${ }^{18}$ ${ }^{20}$ Briefly, in both trials treatment consisted of nine 28-day cycles of KCyD followed by maintenance with single-agent carfilzomib until disease progression or intolerance. Carfilzomib was administered once weekly $\left(70 \mathrm{mg} / \mathrm{m}^{2}\right)$ in the IST-CAR-561 study and twice weekly $\left(36 \mathrm{mg} / \mathrm{m}^{2}\right)$ in the IST-CAR-506 study. The same doses and schedules of cyclophosphamide (oral $300 \mathrm{mg}$ on days 1, 8 and 15) and dexamethasone (40 mg on days 1, 8, 15 and 22) were used in both studies.

\section{Endpoints}

The aim of our analysis was to compare treatment efficacy, in terms of response to therapy, PFS, PFS-2 and OS in patients with SR versus HiR cytogenetics receiving carfilzomib-based regimens.

Cytogenetic risk was centrally assessed by FISH analysis and $t(4 ; 14), t(11 ; 14), t(14 ; 16)$, del13 and del17p were evaluated in both studies. A $15 \%$ cut-off point was used for detection of translocations and a $10 \%$ cut-off point for deletions. FISH analysis was performed on $\mathrm{CD}_{138^{+}}$purified plasma cells. According to the Revised International Staging System (R-ISS) criteria proposed by the International Myeloma Working Group (IMWG) in 2015 , high cytogenetic risk was defined by the presence of at least one CA among del17p, $\mathrm{t}(4 ; 14)$ or $\mathrm{t}(14 ; 16) .{ }^{21}$ Patients' fitness was defined according to the IMWG frailty score, ${ }^{2}$ and patients were classified as either fit, intermediate fit or unfit.

\section{Statistical analysis}

Data from the two trials were pooled together and analyzed. Comparisons between different patient groups were performed using Fisher's exact test. PFS was calculated from the date of enrollment to the date of progression or death, or the date the patient was last known to be in remission. PFS- 2 was calculated from the date of enrollment to the date of second relapse/progression or death or the date the patient was last known to be in remission. OS was calculated from the date of enrollment to the date of death or the date the patient was last known to be alive.

Time-to-event data were analyzed using the Kaplan-Meier method; survival curves were compared with the log-rank test. The Cox proportional hazards model was used to estimate the hazard ratio (HR) values and the $95 \%$ confidence intervals (CI). All reported $P$-values were two-sided at the conventional 5\% significance level. In order to account for potential confounders, the comparison SR versus HiR was adjusted for age, International Staging System (ISS), IMWG Frailty Score and trial (once- vs. twice-weekly carfilzomib).

Data were analyzed using R software (version 3.5.1). 
Table 1. Patients' characteristics at baseline.

\begin{tabular}{|c|c|c|c|}
\hline & $\begin{array}{l}\text { All patients } \\
n=94\end{array}$ & $\begin{array}{l}\text { Standard-risk patients } \\
\qquad \mathrm{n}=57\end{array}$ & $\begin{array}{c}\text { High-risk patients } \\
n=37\end{array}$ \\
\hline \multicolumn{4}{|l|}{ Age } \\
\hline Median (range) & $72(68-75)$ & $72(68-75)$ & $72(68-74)$ \\
\hline$\geq 75$ years, $n(\%)$ & $24(26 \%)$ & $16(28 \%)$ & $8(22 \%)$ \\
\hline \multicolumn{4}{|l|}{ Sex, n (\%) } \\
\hline Male & $40(43 \%)$ & $24(42 \%)$ & $16(43 \%)$ \\
\hline Female & $54(57 \%)$ & $33(58 \%)$ & $21(57 \%)$ \\
\hline \multicolumn{4}{|l|}{ ISS, n (\%) } \\
\hline I & $28(30 \%)$ & $19(33 \%)$ & $9(24 \%)$ \\
\hline II & $32(34 \%)$ & $17(30 \%)$ & $15(41 \%)$ \\
\hline III & $34(36 \%)$ & $21(37 \%)$ & $13(35 \%)$ \\
\hline \multicolumn{4}{|l|}{ FISH, n (\%) } \\
\hline $\mathrm{t}(4 ; 14)$ & $12(13 \%)$ & - & $12(32 \%)$ \\
\hline $\mathrm{t}(14 ; 16)$ & $4(4 \%)$ & - & $4(11 \%)$ \\
\hline del17p & $22(23 \%)$ & - & $22(59 \%)$ \\
\hline$\geq 2 \mathrm{CA}^{*}$ & $1(1 \%)$ & - & $1(3 \%)$ \\
\hline \multicolumn{4}{|l|}{ Frailty Score, n (\%) } \\
\hline Fit & $53(56 \%)$ & $34(60 \%)$ & $19(51 \%)$ \\
\hline Intermediate & $29(31 \%)$ & $18(32 \%)$ & $11(30 \%)$ \\
\hline Frail & $12(13 \%)$ & $5(9 \%)$ & $7(19 \%)$ \\
\hline \multicolumn{4}{|l|}{ LDH [UI/mmol] } \\
\hline Median (range) & $282.5(168-361)$ & 288 (198-359) & 274 (154-386) \\
\hline Missing & $18(19 \%)$ & $13(23 \%)$ & $5(14 \%)$ \\
\hline
\end{tabular}

ISS: International Staging System; FISH: fluorescence in situ hybridization; LDH: lactate dehydrogenase; n: number; CA: cytogenetic abnormalities. *At least two cytogenetic abnormalities among $\mathrm{t}(4 ; 14), \mathrm{t}(14 ; 16)$ and del17p.

\section{Results}

Among the 121 patients enrolled in the two trials (63 patients from IST-CAR-561 and 58 patients from ISTCAR-506), complete cytogenetic data were available for 94 patients: 57 patients $(61 \%)$ in the SR and $37(39 \%)$ in the HiR group according to FISH analysis. Among patients in the HiR group, $t(4 ; 14)$ was present in 12 patients $(13 \%)$, $t(14 ; 16)$ in four patients $(4 \%)$, and del17p in $22(23 \%)$ patients. The median percentage of plasma cells with $\mathrm{t}(4 ; 14)$ was $80 \%$ (range: $15-99)$, with $\mathrm{t}(14 ; 16)$ was $85 \%$, and with del17p was 34\% (range: 10-95).

Baseline characteristics were well balanced between SR and $\mathrm{HiR}$ patients and are summarized in Table 1. Median age at enrollment was 72 years (range: 60-86) for the entire population; no significant differences in terms of age, sex, ISS stage or frailty status were observed between the two groups.

Median follow-up was 38 months for the entire cohort. Ninety-two of 94 patients started the induction phase (1 withdrew consent and 1 was lost to follow-up before commencing therapy): 56 of 57 in the SR and 36 of 37 in the HiR group. Seventy patients $(74 \%)$ started the maintenance phase: $42(74 \%)$ in the SR and $28(76 \%)$ in the HR group $(P=1.00)$. The median duration of treatment was 16.9 months in SR patients and 14.6 months in HiR patients.

Responses to therapy are shown in Table 2. No significant differences in terms of overall response rate (ORR) were observed between SR and HiR patients both after the induction phase ( $86 \%$ and $92 \%$, respectively; $P=0.52)$ and overall (induction and maintenance phases; $88 \%$ and $95 \%$, respectively; $P=0.47$ ). In addition, the rate of complete response (CR) after the induction phase $(19 \%$ vs. $22 \% ; P=0.80)$ and the maintenance phase $(23 \%$ vs. $24 \%$; $P=1$ ) was similar in SR and in HiR patients.

Median PFS was similar between SR (not reached [NR]) and HiR (27.8 months) patients (HR 0.81, 95\%CI: 0.44$1.48 ; P=0.50)$; at 3 years, $52 \%$ and $43 \%$ of patients were alive and free from progression in the two groups, respectively. Median PFS-2 was NR and 44.1 months, respectively (HR 0.67, 95\%CI: 0.32-1.39; $P=0.28$ ). No significant differences were observed in median OS in SR and HiR patients, respectively (median OS: NR vs. NR, HR 0.72, $95 \%$ CI: $0.34-1.52 ; P=0.38$ ), with $78 \%$ of patients in the SR and $73 \%$ in the HiR group alive at 3 years from diagnosis (Figure 1A-C).

No significant differences in terms of median PFS, PFS-2 and OS were observed among patients with or without del17p (PFS: 35 vs. 35.7 months, HR 0.92, 95\%CI: 0.471.82, $P=0.82$; PFS-2: 44.1 months vs. NR, HR $1.20,95 \% \mathrm{CI}$ : $0.55-2.64, P=0.65$; OS: 47.5 months vs. NR, HR $1.17,95 \%$ CI: 0.52-2.62, $P=0.70$ ) (Figure 2). When adopting a higher cut-off for del17p positivity $(>20 \%)$, no significant difference in PFS was reported between del17p-negative and del17p-positive patients (median: 35.7 vs. 35 months).

\section{Discussion}

The aim of our analysis was to evaluate whether a carfilzomib-based upfront treatment could abrogate the 


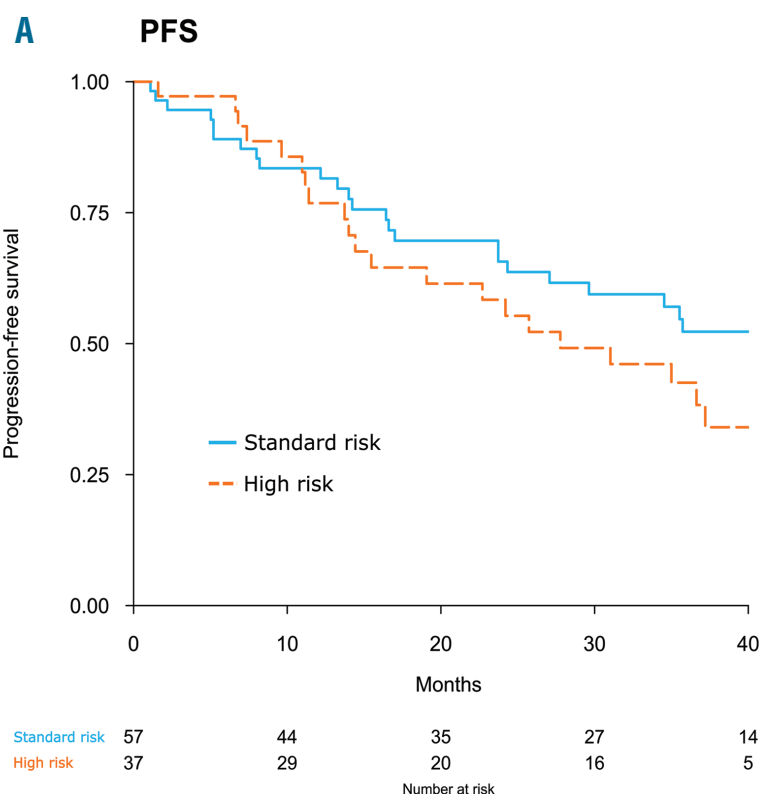

C OS

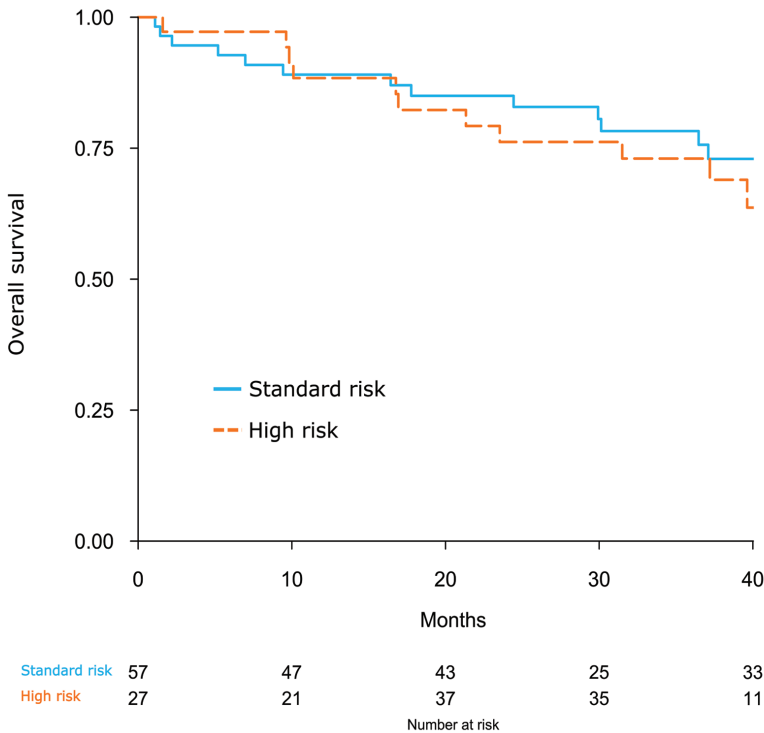

negative impact of HiR cytogenetics and ameliorate the prognosis of transplant-ineligible MM patients carrying HiR CA.

Our results showed similar ORR and CR/stringent CR rates between SR and HiR patients according to the cytogenetic profile, as well as no significant differences in terms of PFS, PFS-2 and OS between the two groups. Furthermore, KCyd seemed to mitigate the poor prognosis conferred by del17p in terms of PFS, PFS-2 and OS.

In Europe, Rd and VMP are currently the first-line regimens of choice for the treatment of older NDMM patients. To date, however, no prospective data on the comparison of VMP and Rd have been published, and the results of the first prospective, phase IV trial comparing these two standards of care are awaited (clinicaltrials.gov identifier: NCT03829371). However, we have recently published a pooled analysis of two phase III studies in which patients were treated either with VMP or Rd plus lenalidomide maintenance (Rd-R), showing a PFS (HR: 0.54) and

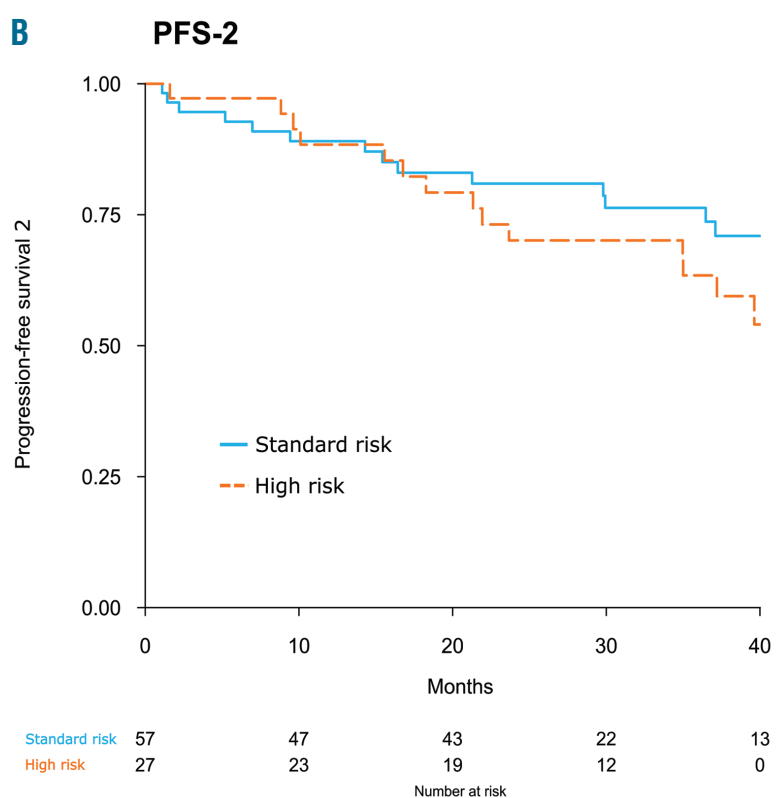

Figure 1. Standard-risk versus high-risk patients. (A) Progression-free surviva (PFS), (B) PFS-2, and (C) overall survival (OS).

OS (HR: 0.73) advantage in $\mathrm{HiR}$ patients receiving bortezomib upfront. ${ }^{22}$ These results were in line with those generated in another phase III study in the transplant setting, in which bortezomib partially improved the poor prognosis of HiR patients carrying $\mathrm{t}(4 ; 14)$ and/or del17p..$^{23}$

In the ASPIRE trial, the addition of carfilzomib to $\mathrm{Rd}$ (KRd) improved the median PFS of approximatively 10 months compared to $\mathrm{Rd}$ in patients with $\mathrm{HiR}$ cytogenetics, although median PFS in HiR patients treated with KRd (23 months) remained approximatively 6 months shorter than in SR patients (29 months). ${ }^{17}$ In the ENDEAVOR trial, the doublet $\mathrm{Kd}$ proved to be superior to $\mathrm{Vd}$ in $\mathrm{HiR}$ patients (HR for PFS: 0.64, 95\% CI: 0.45-0.92; $P=0.007$ ), although median PFS was inferior in HiR versus SR patients receiving $\mathrm{Kd}$ (8.8 months vs. NR, respectively).$^{16}$ In HiR RRMM patients, ixazomib in combination with $\mathrm{Rd}$ also proved to be effective as compared to Rd (HR 0.54, 95\% CI: 0.320.91; $P=0.021$, with similar median PFS in HiR and SR patients treated with this triplet (21.4 and 20.6 months, 
Table 2. Best response after induction phase and overall (induction and maintenance).

\begin{tabular}{|c|c|c|c|}
\hline & $\begin{array}{c}\text { All patients } \\
n=94\end{array}$ & $\begin{array}{l}\text { Standard-risk patients } \\
n=57\end{array}$ & $\begin{array}{c}\text { High-risk patients } \\
n=37\end{array}$ \\
\hline \multicolumn{4}{|c|}{ Response after induction } \\
\hline ORR, n (\%) & $83(88 \%)$ & $49(86 \%)$ & $34(92 \%)$ \\
\hline $\mathrm{sCR} / \mathrm{CR}$ & $19(20 \%)$ & $11(19 \%)$ & $8(22 \%)$ \\
\hline VGPR & $42(45 \%)$ & $25(44 \%)$ & $17(46 \%)$ \\
\hline PR & $22(23 \%)$ & $13(23 \%)$ & $9(24 \%)$ \\
\hline SD & $6(6 \%)$ & $4(7 \%)$ & $2(5 \%)$ \\
\hline NA & $5(5 \%)$ & $4(7 \%)$ & $1(3 \%)$ \\
\hline \multicolumn{4}{|c|}{ Response, induction and maintenance } \\
\hline ORR, n (\%) & $85(90 \%)$ & $50(88 \%)$ & $35(95 \%)$ \\
\hline sCR/CR & $22(23 \%)$ & $13(23 \%)$ & $9(24 \%)$ \\
\hline VGPR & $42(45 \%)$ & $25(44 \%)$ & $17(46 \%)$ \\
\hline PR & $21(22 \%)$ & $12(21 \%)$ & $9(24 \%)$ \\
\hline SD & $4(4 \%)$ & $3(5 \%)$ & $1(3 \%)$ \\
\hline $\mathrm{NA}$ & $5(5 \%)$ & $4(7 \%)$ & $1(3 \%)$ \\
\hline
\end{tabular}

ORR: overall response rate; CR: complete response; sCR: stringent CR;VGPR: very good partial response; PR: partial response; SD: stable disease; NA: not available; n: number.

respectively). ${ }^{24}$ The efficacy of newer $\mathrm{PI}$ in HiR patients may be even more pronounced in the upfront setting, in which the probability of HiR patients treated with KRd of achieving at least a very good partial response ( $\geq V G P R$ ) or a CR was similar to that of SR patients..$^{25}$ In the phase II FORTE study, similar $\geq$ VGPR rates $(79 \%$ vs. $86 \%)$ and minimal residual disease negativity ( $62 \%$ vs. $49 \%)$ were obtained with eight cycles of KRd irrespective of ASCT in both SR and HiR disease according to the R-ISS. ${ }^{26}$ These results confirmed the efficacy in HiR patients that we observed with carfilzomib in the non-transplant setting.

The IMWG recommends the inclusion of a PI in the upfront treatment of HiR NDMM patients. ${ }^{21}$ Our results are in line with the evidence that PI, especially those of the second generation such as carfilzomib, can at least partially abrogate the adverse impact of high-risk CA and ameliorate the prognosis of HiR patients.

As we mentioned above, current approved treatment options in transplant-ineligible NDMM patients include Rd, VMP with or without daratumumab and VRD, with Dara-Rd coming soon. Despite the pitfalls of cross-trial comparisons, the median PFS and OS observed in HiR patients receiving carfilzomib-based therapy in our analysis compare favorably with those observed in HiR patients receiving $\mathrm{Rd}$ in the FIRST trial ${ }^{15}$ (PFS: 8.4 months; OS: 29.3 months) and VMP in the VISTA study ${ }^{12}$ (median PFS: 19.8 months), with results similar to those observed in HiR patients treated with Dara-Rd in the phase III MAIA study. ${ }^{27}$ Daratumumab, combined to either VMP or Rd, will represent the new standard of care in the upfront treatment of patients ineligible for transplant. The median PFS of patients treated with Dara-VMP was 36.4 months in the recently updated ALCYONE study and NR at 30 months in the MAIA study with Dara-Rd. ${ }^{28,29}$ Despite these impressive results, the PFS benefit seemed striking in SR patients (HR 0.39 for Dara-VMP and 0.49 for Dara-Rd), while it was less evident in HiR patients (HR 0.78 for Dara-VMP and 0.85 for Dara-Rd). In the era of anti-CD38based first-line regimens, HiR genetic lesions are still an unfavorable prognostic factor and HiR patients continue to represent an unmet medical need.

Our analysis has some limitations. First of all, the small

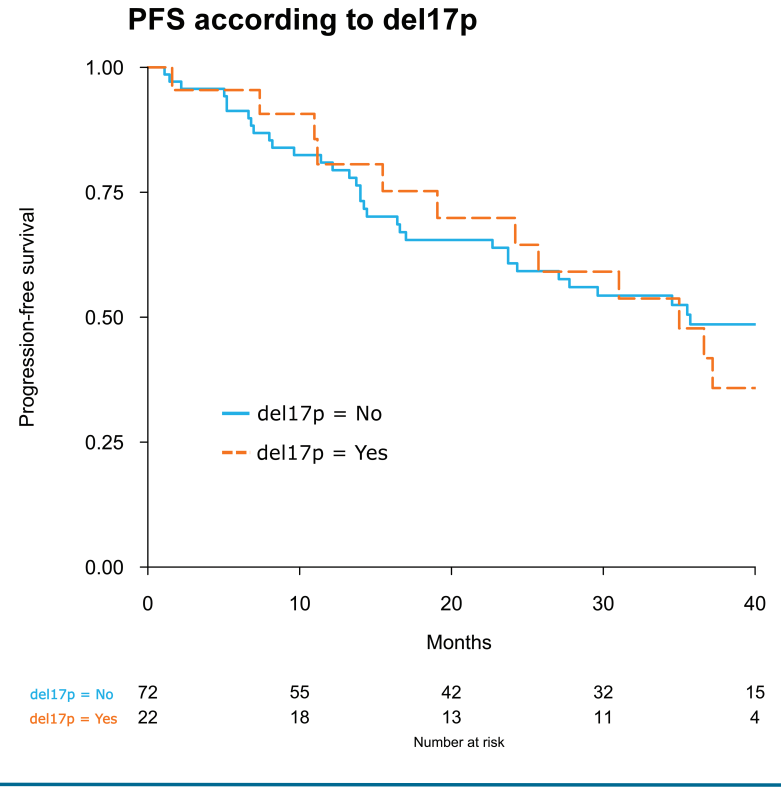

Figure 2. Median progression-free survival (PFS) according to del17p status.

number of patients analyzed does not allow definite conclusions to be drawn on this issue, but prompts further evaluation of carfilzomib as induction therapy in transplant-ineligible patients. We used a $10 \%$ cut-off to define the positivity or negativity for del17p, even though the median percentage of plasma cells with del17p was slightly higher (34\%; range: $17-80)$. The exact cut-off to be used to define del17p positivity is a matter of controversy. While the Mayo Clinic group showed no correlation between PFS and OS and the mutational burden in del17p patients, a recent study published by Thakurta et al. showed a positive correlation between a high cancer clonal fraction and survival outcomes. ${ }^{30,31}$ Remarkably, our results remained consistent when a higher cut-off for del17p positivity was adopted $(>20 \%$, as in the ENDEAVOR trial). At the same time as the two trials included in our analysis were being designed, the impact of other HiR 
genetic features, such as bi-allelic inactivation, was still unknown, and therefore it could not be addressed in our work.

The prolonged use of carfilzomib in our study may have had a beneficial role in HiR patients. The available evidence suggests that continuous therapy could be superior to fixed duration therapy and could be of particular benefit to HiR patients. However, continuous therapy is not sufficient to overcome the poor prognosis of adverse CA. For example, in the FIRST study, the median PFS of HiR patients treated with continuous $\mathrm{Rd}$ was only 9 months. ${ }^{14,15}$ In our analysis, the median duration of therapy was similar between SR and HiR patients (16.9 vs. 14.6 months), meaning that both groups of patients benefited from prolonged treatment. In conclusion, the results of our pooled analysis suggest that a carfilzomib-based treatment is effective as upfront treatment for HiR, transplantineligible MM patients. Carfilzomib may contribute to fill the gap between SR and HiR patients, thus improving the poor prognosis of the latter. Our results provide the basis for a further investigation of carfilzomib as upfront therapy for the treatment of HiR MM patients.

\section{Disclosures}

$R M$ has received honoraria from Sanofi, Celgene, Takeda and Janssen; has served on the advisory boards for Sanofi, Takeda, and Janssen; has received consultancy fees from Janssen; MTP has received honoraria from Celgene, JanssenCilag, BMS, Takeda, and Amgen, has served on the advisory boards for Celgene, Janssen-Cilag, BMS, Takeda, and Amgen; $A M L$ has received honoraria from Janssen, Celgene, BristolMyers Squibb, and Servier, has received clinical trial support from Novartis, AbbVie, Roche, Amgen, and Celgene, has served on the advisory boards for AbbVie, Amgen, Takeda, and Servier, and has undertaken consultancy for Incyte; StB has received honoraria for attending meetings from Janssen and Celgene; PM has received personal fees from Amgen, Novartis, BMS, Celgene, Janssen, and Takeda; GB has served on the advisory boards for Novartis, Celgene, and Amgen; MC has received grants from Janssen and Celgene; has received personal fees from Janssen, Celgene, Amgen, BMS, and Takeda; AP is currently a GlaxoSmithKline AG employee; VM has received speaking fees from and served on the advisory boards for Amgen, Celgene, Janssen, and Takeda; GG has served on the advisory boards for Janssen, AbbVie, Astra-Zeneca, and Sunesys, has served on the speakers' bureaus for Janssen,
Gilead, and AbbVie; PO has served on the advisory boards for Janssen; $M B$ has received honoraria from Sanofi, Celgene, Amgen, Janssen, Novartis, Bristol-Myers Squibb, and AbbVie; has served on the advisory boards for Janssen and GSK; has received research funding from Sanofi, Celgene, Amgen, Janssen, Novartis, Bristol-Myers Squibb, and Mundipharma; $S B$ has received honoraria from Bristol-Myers Squibb, Celgene, Amgen and Janssen, has served on the advisory boards for Amgen, Karyopharm, Janssen and Celgene, and has received consultancy fees from Takeda and Janssen. The remaining authors have no conflicts of interest to disclose.

\section{Contributions}

$R M, F B, P O, M B$ and $S B$ made substantial contributions to the conception or design of the analysis; all authors are responsible for the acquisition, analysis or interpretation of data; $R M, F B$, $A C, M G, P O$ and $S B$ made the first draft of the manuscript; $A C$ carried out the statistical analysis; $M B$ and $S B$ supervised the analysis; all authors critically revised the manuscript for important intellectual content; all authors gave their final approval of the version to be published; all authors agree to be accountable for all aspects of the work in ensuring that questions related to the accuracy or integrity of any part of the work are appropriately investigated and resolved.

\section{Acknowledgments}

We thank the patients who took part in these studies and their families, the study co-investigators, nurses and co-ordinators at each of the clinical sites. We would also like to thank the nurses Rosalia Capobianco and Giacomo Castorina, and the data managers Debora Caldarazzo and Federica Leotta.

\section{Funding}

The IST-CAR-561 (NCT01857115) study was sponsored by Stichting Hemato-Oncologie voor Volwassenen Nederland (HOVON, the Netherlands), in collaboration with Fondazione Neoplasie Sangue ONLUS (Italy). The IST-CAR-506 (NCT01346787) study was sponsored by the HOVON Foundation and co-sponsored by Fondazione Neoplasie Sangue ONLUS. Both trials were supported by funding from AMGEN (Onyx Pharmaceuticals), which had no role in study design, data collection, data analysis, data interpretation, writing of the report or publication of this article. The corresponding author had full access to all the data in the two studies, and had final responsibility for the decision to prepare and submit this manuscript for publication, together with the other authors.

\section{References}

1. Greipp PR, San Miguel J, Durie BGM, et al. International staging system for multiple myeloma. J Clin Oncol. 2005;23(15):34123420.

2. Palumbo A, Bringhen S, Mateos M-V, et al. Geriatric assessment predicts survival and toxicities in elderly myeloma patients: an International Myeloma Working Group report. Blood. 2015;125(13):2068-2074

3. Larocca A, Dold SM, Zweegman S, et al. Patient-centered practice in elderly myeloma patients: an overview and consensus from the European Myeloma Network (EMN). Leukemia. 2018;32(8):1697-1712.

4. Fonseca R, Bergsagel PL, Drach J, et al. International Myeloma Working Group molecular classification of multiple myelo- ma: spotlight review. Leukemia. 2009; 23(12):2210-2221.

5. Moreau P, Attal M, Garban F, et al. Heterogeneity of $\mathrm{t}(4 ; 14)$ in multiple myeloma. Long-term follow-up of 100 cases treated with tandem transplantation in IFM99 trials. Leukemia. 2007;21(9):2020-2024.

6. Gertz MA, Lacy MQ, Dispenzieri A, et al. Clinical implications of $t(11 ; 14)(q 13 ; q 32)$, $\mathrm{t}(4 ; 14)(\mathrm{p} 16.3 ; \mathrm{q} 32)$, and $-17 \mathrm{p} 13$ in myeloma patients treated with high-dose therapy. Blood. 2005;106(8):2837-2840.

7. Fonseca R, Blood E, Rue M, et al. Clinical and biologic implications of recurrent genomic aberrations in myeloma. Blood. 2003;101(11):4569-4575.

8. Avet-Loiseau H, Malard F, Campion L, et al. Translocation $\mathrm{t}(14 ; 16)$ and multiple myeloma: is it really an independent prognostic factor? Blood. 2011;117(6):2009-2011.
9. Palumbo A, Avet-Loiseau H, Oliva S, et al. Revised International Staging System for Multiple Myeloma: a report from International Myeloma Working Group. J Clin Oncol. 2015:33(26):2863-2869.

10. Howlader N, Noone A, Krapcho M, et al. Cancer Statistics Review, 1975-2015, National Cancer Institute. Based on November 2018 SEER data submission, posted to the SEER web site, April 2019 https://seer.cancer.gov/csr/1975_2015/ [Last accessed 7 Jan 2019]

11. Moreau P, San Miguel J, Sonneveld P, et al. Multiple myeloma: ESMO Clinical Practice Guidelines for Diagnosis, Treatment and Follow-up. Ann Oncol. 2017;28(Suppl 4):iv52-iv61.

12. San-Miguel JF, Schlag R, Khuageva NK, et al. Bortezomib plus melphalan and prednisone for initial treatment of multiple myeloma. $\mathrm{N}$ 
Engl J Med. 2008;359(9):906-917.

13. San-Miguel JF, Schlag R, Khuageva NK, et al. Persistent overall survival benefit and no increased risk of second malignancies with bortezomib-melphalan-prednisone versus melphalan-prednisone in patients with previously untreated multiple myeloma. J Clin Oncol. 2013;31(4):448-455.

14. Benboubker L, Dimopoulos MA, Dispenzieri A, et al. Lenalidomide and dexamethasone in transplant-ineligible patients with myeloma. N Engl J Med. 2014; 371(10):906-917.

15. Facon T, Dimopoulos MA, Dispenzieri A, et al. Final analysis of survival outcomes in the phase 3 FIRST trial of up-front treatment for multiple myeloma. Blood. 2018;131(3):301310.

16. Chng W-J, Goldschmidt H, Dimopoulos $\mathrm{MA}$, et al. Carfilzomib-dexamethasone vs bortezomib-dexamethasone in relapsed or refractory multiple myeloma by cytogenetic risk in the phase 3 study ENDEAVOR. Leukemia. 2017;31(6):1368-1374.

17. Avet-Loiseau H, Fonseca R, Siegel D, et al. Carfilzomib significantly improves the progression-free survival of high-risk patients in multiple myeloma. Blood. 2016;128(9):11741180.

18. Bringhen S, Petrucci MT, Larocca A, et al. Carfilzomib, cyclophosphamide, and dexamethasone in patients with newly diagnosed multiple myeloma: a multicenter, phase 2 study. Blood. 2014;124(1):63-69.

19. Bringhen S, D'Agostino M, De Paoli L, et al. Phase $1 / 2$ study of weekly carfilzomib, cyclophosphamide, dexamethasone in newly diagnosed transplant-ineligible myeloma. Leukemia. 2018;32(4):979-985.

20. Bringhen S, Mina R, Petrucci MT, et al. Once-weekly versus twice-weekly carfilzomib in patients with newly diagnosed multiple myeloma: a pooled analysis of two phase I/II studies. Haematologica. 2019; 104(8):1640-1647.

21. Sonneveld P, Avet-Loiseau H, Lonial S, et al. Treatment of multiple myeloma with high risk cytogenetics: a consensus of the International Myeloma Working Group. Blood. 2016;127(24):2955-2962

22. Larocca A, Mina R, Offidani M, et al. Firstline therapy with either bortezomib-melphalan-prednisone or lenalidomide-dexamethasone followed by lenalidomide for transplant-ineligible multiple myeloma patients: a pooled analysis of two randomized trials. Haematologica. 2020; 105(4):1074-1080

23. Sonneveld P, Goldschmidt H, Rosiñol L, et al. Bortezomib-based versus nonbortezomib-based induction treatment before autologous stem-cell transplantation in patients with previously untreated multiple myeloma: a meta-analysis of phase III randomized, controlled trials. J Clin Oncol. 2013;31(26):3279-3287.

24. Avet-Loiseau H, Bahlis NJ, Chng W-J, et al. Ixazomib significantly prolongs progressionfree survival in high-risk relapsed/refractory myeloma patients. Blood. 2017; 130(24):2610-2618.

25. Gay F, Cerrato C, Scalabrini DR, et al Carfilzomib-lenalidomide-dexamethasone (KRd) induction-autologous transplant (ASCT)-Krd consolidation vs KRd 12 cycles vs carfilzomib-cyclophosphamide- dexamethasone (KCd) induction-ASCT$\mathrm{KCd}$ consolidation: analysis of the randomized FORTE trial in newly diagnosed multiple myeloma (NDMM). Blood. 2018;132(Suppl 1):Abstract \#121 [ASH 2018 60th Meeting].

26. Gay F, Rota Scalabrini D, Belotti A, et al. Updated efficacy and MRD data according to risk status in newly diagnosed myeloma patients treated with carfilzomib plus lenalidomide or cyclophosphamide: results from the FORTE trial. HemaSphere. 2018;2(S1):6 [Abstract \#S109, EHA 2018 23rd Congress].

27. Facon T, Kumar SK, Plesner T, et al. Phase 3 randomized study of daratumumab plus lenalidomide and dexamethasone (D-Rd) versus lenalidomide and dexamethasone (Rd) in patients with newly diagnosed multiple myeloma (NDMM) ineligible for transplant (MAIA). Blood. 2018;132(Suppl 1):Abstract \#LBA-2 [ASH 2018 60th Meeting]

28. Facon T, Kumar S, Plesner $T$, et al. Daratumumab plus lenalidomide and dexamethasone for untreated myeloma. N Eng J Med. 2019;380(22):2104-2115.

29. Mateos M-V, Dimopoulos MA, Cavo M et al. Daratumumab plus bortezomib, melphalan, and prednisone for untreated myeloma. N Engl J Med. 2018;378(6):518-528.

30. Lakshman A, Painuly U, Rajkumar SV, et al. Impact of acquired del(17p) in multiple myeloma. Blood Adv. 2019:3(13):1930-1938.

31. Thakurta A, Ortiz M, Blecua P, et al. High subclonal fraction of $17 p$ deletion is associated with poor prognosis in multiple myeloma. Blood. 2019;133(11):1217-1221 